J. Lake Sci. (湖泊科学), 2005, 17(1): 47-53

ISSN 1003 -5427; E-mail: jlakes@ niglas. ac.cn

Copyright 2005 by Journal of Lake Sciences

\title{
湖泊沉积物中元素相态的连续提取分析一一岱海为例 ${ }^{*}$
}

\author{
金章东 ${ }^{1}$, 邹成娟 ${ }^{2}$, 李福春 ${ }^{3}$, 王苏民 ${ }^{1}$, 沈 吉 $^{1}$, 梁文波 ${ }^{2}$ \\ (1: 中国科学院南京地理与湖泊研究所湖泊沉积与环境重点实验室, 南京 210008) \\ $(2$ : 江苏石油勘探局物探技术研究院,南京 210046) \\ ( 3 : 南京农业大学资源与环境科学学院,南京 210095 )
}

摘 要: 如何利用湖泊/海洋沉积物地球化学代用指标有效地指示环境很大程度取决于元素的来源及其在沉积物中赋存 形态的确定. 对位于半干旱区的内蒙古岱海 14 个沉积物样品的连续提取实验及 18 个元素在水可溶态、可交换态、碳酸盐 结合态、铁锰氧化物结合态、有机物结合态及残留态等六种赋存相态中含量变化进行了测试和研究, 结合沉积物的矿物组 成, 结果表明在岱海流域内只经历了较弱的化学风化过程, 除了 $\mathrm{Ca}$ 和 $\mathrm{Sr}$ 外, 其他元素主要赋存于残留态中, 只发生了很 小程度的活化溶解. 受元素亲合性和湖水物理化学状态的制约, 出溶的 $\mathrm{Sr}$ 与 $\mathrm{Ca}$ 的赋存相态基本一致, 而出溶的 $\mathrm{Pb} 、 \mathrm{Be} 、$ $\mathrm{Co} 、 \mathrm{Cu} 、 \mathrm{~K} 、 \mathrm{Mn} 、 \mathrm{Fe}$ 等元素则与自生碳酸盐、有机质表现出一定的亲合性. 元素在不同赋存相态中分布为地球化学环境代用 指标的提取和解释提供了机理性的实验依据.

关键词: 赋存相态; 化学风化; 表生行为;元素;岱海

\section{Element Distribution in Chemical Fractions of Lake Sediments Determined by Sequential Extraction: the Case of Lake Daihai (Northern China)}

\author{
JIN Zhangdong ${ }^{1}$, ZOU Chengjuan ${ }^{2}$, LI Fuchun ${ }^{3}$, WANG Sumin $^{1}{ }^{1}$, SHEN Ji ${ }^{1} \&$ LIANG Wenbo ${ }^{2}$
}

( 1 : Key Laboratory of Lake Sedimentation and Environment, Nanjing Institute of Geography \& Limnology, CAS, Nanjing 210008, P. R. China)

( 2 : Jiangsu Petroleum Exploration Bureau, Nanjing 210046, P. R. China)

(3:School of Resource and Environment Sciences, Nanjing Agriculture University, Nanjïn 210095,P. R. China)

\begin{abstract}
Environmental interpretation of geochemical proxies of lake/ocean sediments largely depends on an understanding of where elements are from and of how elements bound to various fractions in the sediments. Fourteen samples from Lake Daihai, Inner Mongolia, located in semiarid zone, were carried out a sequential extraction experiment and analyzed eighteen elements concentration by ICP-AES or AAS in each chemical fractions defined as follows: soluble, exchangeable, bound to carbonate, bound to Fe-Mn-oxides, bound to organic matter and residual. Our results indicate that weak chemical weathering was proceeded under climate conditions in the Lake Daihai watershed, resulting that most of the elements determined are in residual due to little activation during weathering, except $\mathrm{Ca}$ and $\mathrm{Sr}$, by combining with detrital minerals of predominantly plagioclase. Meanwhile, controlled by element affinity and lake water physicochemical conditions, leached both $\mathrm{Sr}$ and Ca are in the same fractions with good correlation, whereas other activated elements including $\mathrm{Pb}, \mathrm{Be}, \mathrm{Co}, \mathrm{Cu}, \mathrm{K}, \mathrm{Mn}, \mathrm{Fe}$ etc are predominantly associated with the fractions of bound to carbonate and/or organic matter. Consequently, element distribution in various chemical fractions provides a useful experimental evidence for selection and interpretation of some geochemical proxies.
\end{abstract}

Keywords: Chemical fraction; weathering; geochemical behavior at the Earth's surface; element; Lake Daihai

在利用湖泊或海洋沉积物对古环境、古气候进行反演并解释过程中,往往涉及到沉积物中各类元素的 赋存形式或结合状态 ${ }^{[1]}$. 然而, 以往对于沉积物、土壤及水体等体系的地球化学研究, 大多数是在分析元素 总量后根据元素或元素比值解释环境演化的, 而对各元素赋存状态及迁移形式等地球化学特征缺乏机理性 
的深入研究, 这对环境演化的解释具有模糊性和笼统性. 特别是在利用湖泊沉积物进行古环境、古气候演化 研究中,元素赋存状态不仅受源区气候变化、岩性的控制,而且还受水体物理-化学状态甚至生物作用的制 约,因此造成很多地球化学环境代用指标的相对性和多解性.

在地球表生作用过程中,流域内高温高压下形成的岩石在暴露地表后将发生物理和化学反应,矿物的 破碎、溶解、分解导致元素分馏及在沉积物中赋存状态的改变, 并记录流域环境变化信息. 流域内因人类活 动加人到湖盆中的元素也将根据其不同的地球化学性状赋存于不同相态中. 如受风化淋溶程度制约 $\mathrm{Ca} 、 \mathrm{Sr}$ 可赋存于可交换态、碳酸盐结合态、铁锰氧化物结合态、有机物结合态及残留态 (主要为硅酸盐) 中,而碳酸 盐结合态中的 $\mathrm{Sr} / \mathrm{Ca}$ 比值不但与水体 $\mathrm{Sr} / \mathrm{Ca}$ 比值和生物对 $\mathrm{Sr}$ 配分的影响有关, 而且沉积后压实作用也将改 变其比值 ${ }^{[2]}$. 又如沉积物中的 $\mathrm{CaCO}_{3}$ 含量常用作湖盆干湿变化指标, 这在无形中假定了所有形式的 $\mathrm{Ca}$ 对环 境变化具有同等的响应,这显然是站不住脚的,因此常常出现与其他环境指标 (如 $\delta^{13} \mathrm{C}_{\mathrm{org}}$ 、孢粉等) 相矛盾的 现象 ${ }^{[3]}$. 湖泊富营养化对金属元素的赋存相态也有直接的影响 (如 $[4,5]$ ), 如硫化物对沉积物中金属元素 束缚过程起重要作用,而湖泊的富营养化则会减少 $\mathrm{Cu} 、 \mathrm{Ni}$ 在水中的滞留时间. 因此,沉积物中元素赋存相态 关系到各体系中元素迁移、转移、生物可得性的评估以及元素及其同位素组成变化对环境演化正确并真实 地解释.

本文借鉴 Tessier 等 ${ }^{[6]}$ 和 $\mathrm{Li}$ 等 ${ }^{[7]}$ 的思路及其改进后的实验方法, 对内蒙古岱海 $\left(112^{\circ} 32^{\prime} 31^{\prime \prime}-112^{\circ} 48^{\prime}\right.$ $40^{\prime \prime} \mathrm{E}, 40^{\circ} 28^{\prime} 7^{\prime \prime}-40^{\circ} 39^{\prime} 6^{\prime \prime} \mathrm{N}$ ) 一系列沉积物的连续提取实验, 分析元素在水可溶态、可交换态、碳酸盐结合 态、铁锰氧化物结合态、有机物结合态及残留态 (主要为硅酸盐) 等六种相态中的相对含量, 并将之与国际 标准 (USGS MAG - $1^{[8]}$ ) 进行对比, 从而确定不同元素在沉积物中的主要赋存状态. 进而探讨湖泊沉积物中 元素赋存相态与表生风化行为、湖泊矿物沉积的关系, 为一些环境指标 (如 $\mathrm{CaCO}_{3}$ 含量、 $\mathrm{Rb} / \mathrm{Sr}$ 比、 $\mathrm{Sr} / \mathrm{Ca}$ 比 等) 提供地球化学依据,也将为利用沉积物进行特定目的的研究提供相应赋存相的提取方法.

\section{1 连续提取方法}

鉴于对元素赋存状态对其地球化学行为的影响, 目前已有一些实验方法, 主要有两种: 酸溶相-酸不溶 相分离和连续提取法. 前者已被不少研究者所重视并较多运用, 且简单、快速, 大量用于黄土-古土壤的酸溶 实验 ${ }^{[9]}$ 、酸溶相硼的分离及其同位素测定 ${ }^{[10]}$, 但由于这种分离过于笼统, 难以找到一种理想的溶剂使之完 全分离. 连续提取法主要应用于对土壤、沉积物中微量金属及污染源的研究 ${ }^{[6,7]}$, 而较少涉及元素的表生地 球化学行为. 本文选取了岱海沉积岩心中 14 个代表性样品, 参考文献中的实验方法 ${ }^{[6,7]}$ 进行了元素赋存相 态的连续提取分析. 首先, 将样品在室温下干燥并磨至 200 目后, 在 $105^{\circ} \mathrm{C}$ 下烘 $4 \mathrm{~h}$ 以上,准确称取 $1.000 \mathrm{~g}$ 粉末作为实验材料;然后用去离子水和一系列试剂在不同的溶解温度和时间内分别提取水可溶态、可交换 态、碳酸盐结合态、铁锰氧化物结合态、有机物结合态,剩下的归为残留态 (主要为硅酸盐). 每级提取实验 中, 保持溶液体积相同. 将离心后吸取的各级清液加人 $5 \mathrm{ml} \mathrm{HNO}$ 后烘干, 再用稀盐酸和去离子水制成溶 液, 并用去离子水定容至 $10 \mathrm{ml}$. 残留态经微波高温消化后定容至 $100 \mathrm{ml}$, 吸取 $5 \mathrm{ml}$, 加人 $2 \mathrm{ml} 5 \mathrm{~mol} / \mathrm{L}$ 的 $\mathrm{HCl}$, 用去离子水也定容至 $10 \mathrm{ml}$. 为检验提取方法的有效性和精度, 每 7 个样品中至少加人一个重复样. 将 定容后的溶夜在南京大学成矿作用国家重点实验室用 ICP - AES 测定 P、Ni、V、Ca、K、Zn、Mn、Be、Al、Pb、Fe、 $\mathrm{Cu} 、 \mathrm{Sr} 、 \mathrm{Co} 、 \mathrm{Cr} 、 \mathrm{Ti} 、 \mathrm{Li}$ 等元素的含量; 在南京大学现代分析中心用原子吸收法 (AAS) 测定了溶液中 $\mathrm{Rb}$ 的含 量. 同时,采用同样的测定方法分析了 2 个分别采自表层和底层的湖水样品的上述 18 个元素的含量. 误差 一般小于 $5 \%$.

\section{2 结果与讨论}

表 1 中列出了 18 个元素在岱海湖水及 14 个沉积物样品的六种赋存状态中的平均含量,各元素及其在 不同赋存状态中的含量变化在 2 至 5 个数量级之间,并且不同元素主要赋存状态也完全不同. 采自表层和 底层的湖水样品各元素含量基本一致. 根据各元素在沉积物各赋存状态中含量的高低, 将各赋存状态中主 要元素归纳如表 2 所列. 结合表 1 和 2 可以看出, 虽然湖水和沉积物的水可溶态、可交换态中最主要的元素 是 $\mathrm{Ca} 、 \mathrm{~K} 、 \mathrm{Sr}$, 但是碳酸盐结合态具有最高含量的 $\mathrm{Ca} 、 \mathrm{Sr}$, 而最高含量的 $\mathrm{K}$ 出现在残留态中 (表 1 ). 主要赋存 


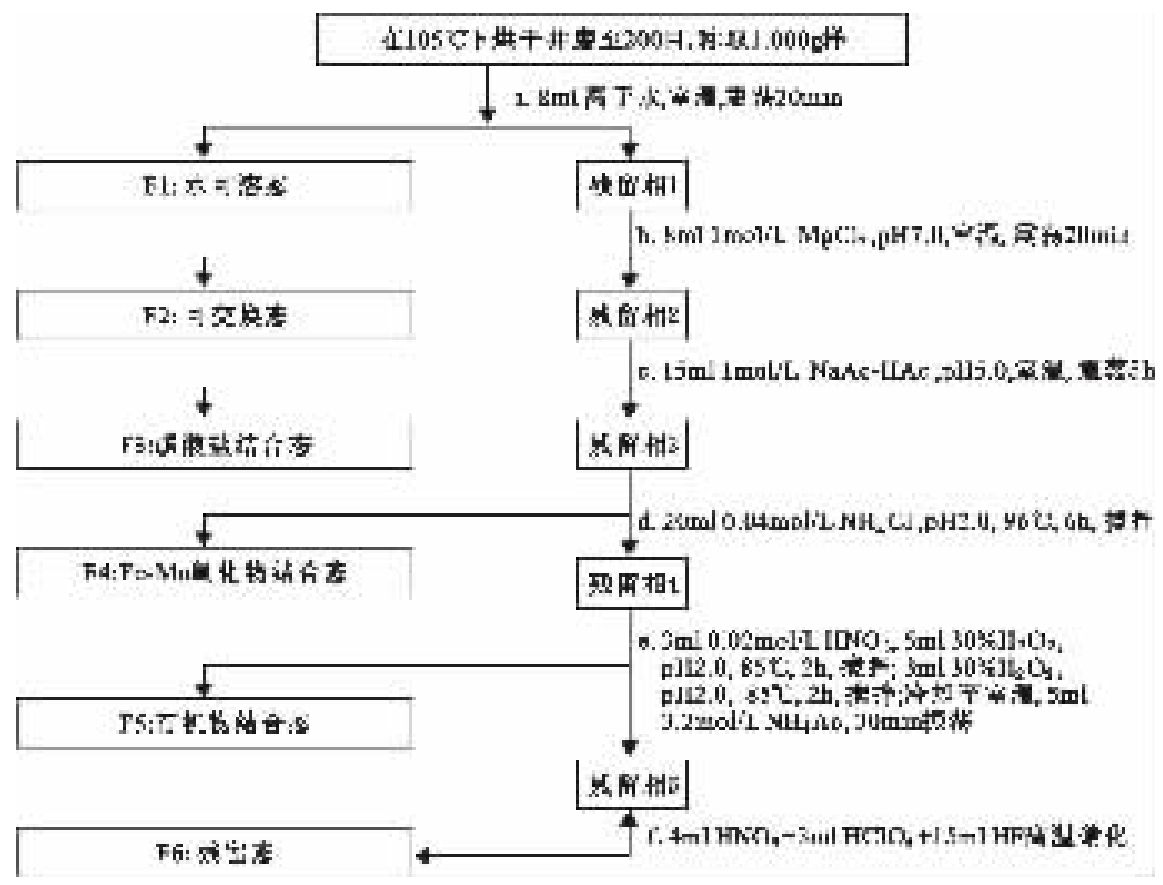

图 1 连续提取实验条件及操作流程简图 (据文献 $[6,7]$ )

Fig. 1 A sketchy scheme of experimental conditions and operation procedure of sequential extraction 表 1 元素在岱海湖水及沉积物各赋存状态中的平均含量 $\left(10^{-9}\right)^{1)}$

Tab. 1 Mean concentrations of some elements in lake water and in six fractions of the Daihai lake sediments $\left(10^{-9}\right)$

\begin{tabular}{cccccccc}
\hline 元素 & $\begin{array}{c}\text { 湖水 } \\
(2)\end{array}$ & $\begin{array}{c}\text { 水可溶态 } \\
(14)\end{array}$ & $\begin{array}{c}\text { 可交换态 } \\
(14)\end{array}$ & $\begin{array}{c}\text { 碳酸盐结合态 } \\
(14)\end{array}$ & $\begin{array}{c}\text { Fe-Mn 氧化物结合态 } \\
(14)\end{array}$ & $\begin{array}{c}\text { 有机物结合态 } \\
(14)\end{array}$ & $\begin{array}{c}\text { 残留态 } \\
(14)\end{array}$ \\
\hline $\mathrm{P}$ & 47.61 & 0.67 & 35.72 & 69.51 & 93.45 & 39.71 & 940.72 \\
$\mathrm{Ni}$ & 8.85 & 0.08 & 13.25 & 31.82 & 16.41 & 7.18 & 41.70 \\
$\mathrm{~V}$ & 46.78 & 0.17 & 552.30 & 53.02 & 47.28 & 5.27 & 113.69 \\
$\mathrm{Ca}$ & 8157 & 136.40 & 2692 & 31669 & 4632 & 1017 & 4336 \\
$\mathrm{~K}$ & 2795 & 68.41 & 2104 & 5058 & 595 & 112 & 20370 \\
$\mathrm{Zn}$ & 2.80 & 0.04 & 3.19 & 10.85 & 25.59 & 5.44 & 90.18 \\
$\mathrm{Mn}$ & 0.91 & 0.05 & 21.92 & 328.56 & 469.15 & 37.92 & 342.84 \\
$\mathrm{Be}$ & 0.07 & - & 0.078 & 0.27 & 0.45 & 0.08 & 1.59 \\
$\mathrm{Al}$ & 75.37 & 1.23 & 19.28 & 97.64 & 2218.99 & 680.24 & 78.80 \\
$\mathrm{~Pb}$ & 34.65 & 0.18 & 59.26 & 139.69 & 24.45 & 22.43 & 82.32 \\
$\mathrm{Fe}$ & 23.01 & 0.44 & 16.83 & 77.91 & 6924 & 1306 & 47947 \\
$\mathrm{Cu}$ & 3.89 & 0.13 & 2.89 & 10.66 & 4.58 & 4.02 & 42.74 \\
$\mathrm{Rb}$ & - & 0.06 & 0.94 & 2.24 & 1.14 & 0.34 & 156.75 \\
$\mathrm{Sr}$ & 167.95 & 1.74 & 34.55 & 222.33 & 189.95 & 7.16 & 113.26 \\
$\mathrm{Co}$ & 2.43 & 0.01 & 2.89 & 6.753 & 2.82 & 1.09 & 8.45 \\
$\mathrm{Cr}$ & 5.37 & 0.04 & 13.08 & 35.870 & 13.225 & 8.40 & 102.70 \\
$\mathrm{Ti}$ & 2.32 & 0.03 & 1.78 & 6.99 & 0.984 & 9.32 & 4620.23 \\
$\mathrm{Li}$ & 4.28 & 0.07 & 1.63 & 7.15 & 9.044 & 1.88 & 56.23 \\
\hline
\end{tabular}

1) 括弧 ()内为样品数; - 表示低于检测值. 
于残留态中的元素还包括 $\mathrm{P} 、 \mathrm{Al} 、 \mathrm{Ti} 、 \mathrm{Rb} 、 \mathrm{Cr} 、 \mathrm{Co} 、 \mathrm{Cu}$ 等元素; 有机物结合态中包含的最主要的元素是 $\mathrm{Fe} 、 \mathrm{~K} 、$ Ca. 由此可见, 岱海沉积物中元素的赋存相态具有很大的差异,这种差异主要受各元素在表生风化过程中的 地球化学行为及岱海流域的气候环境状态制约的.

表 2 岱海沉积物中不同元素的主要赋存状态及制约因素

Tab. 2 dominantchemical fractions and control factors of some elements in the Daihai lake sediments

\begin{tabular}{ll}
\hline 赋存状态 & 元素 \\
\hline 湖水 & $\mathrm{Ca} 、 \mathrm{~K} 、 \mathrm{Sr}$ \\
水可溶态 & $\mathrm{Ca} 、 \mathrm{~K}$ \\
可交换态 & $\mathrm{V} 、 \mathrm{~Pb} 、 \mathrm{Ni} 、 \mathrm{Ca} 、 \mathrm{~K}$ \\
碳酸盐结合态 & $\mathrm{Sr} 、 \mathrm{Ca} 、 \mathrm{~Pb} 、 \mathrm{Be} 、 \mathrm{Cr} 、 \mathrm{Co} 、 \mathrm{Cu} 、 \mathrm{~K} 、 \mathrm{Mn} 、 \mathrm{Ni}$ \\
$\mathrm{Fe}-\mathrm{Mn}$ 氧化物结合态 & $\mathrm{Mn} 、 \mathrm{Sr} 、 \mathrm{Ca} 、 \mathrm{Zn} 、 \mathrm{Fe} 、 \mathrm{Li} 、 \mathrm{Be}$ \\
有机物结合态 & $\mathrm{Fe} 、 \mathrm{~K} 、 \mathrm{Ca}$ \\
残留态 & $\mathrm{P} 、 \mathrm{Al} 、 \mathrm{Ti} 、 \mathrm{Rb} 、 \mathrm{Cr} 、 \mathrm{Co} 、 \mathrm{Cu} 、 \mathrm{~K} 、 \mathrm{Fe} 、 \mathrm{Li} 、 \mathrm{Ni} 、 \mathrm{Zn} 、 \mathrm{~Pb} 、 \mathrm{Mn}$ \\
\hline 风化影响程度 & \\
受风化程度制约的 & $\mathrm{Sr} 、 \mathrm{Ca}$ \\
部分受风化程度制约的 & $\mathrm{V} 、 \mathrm{~K} 、 \mathrm{Zn} 、 \mathrm{Mn} 、 \mathrm{Be} 、 \mathrm{~Pb} 、 \mathrm{Fe} 、 \mathrm{Cu} 、 \mathrm{Co}$ \\
基本不受风化程度制约的 & $\mathrm{P} 、 \mathrm{Li} 、 \mathrm{Cr} 、 \mathrm{Ni}$ \\
完全不受风化程度制约的 & $\mathrm{Ti} 、 \mathrm{Al} 、 \mathrm{Rb}$ \\
\hline
\end{tabular}
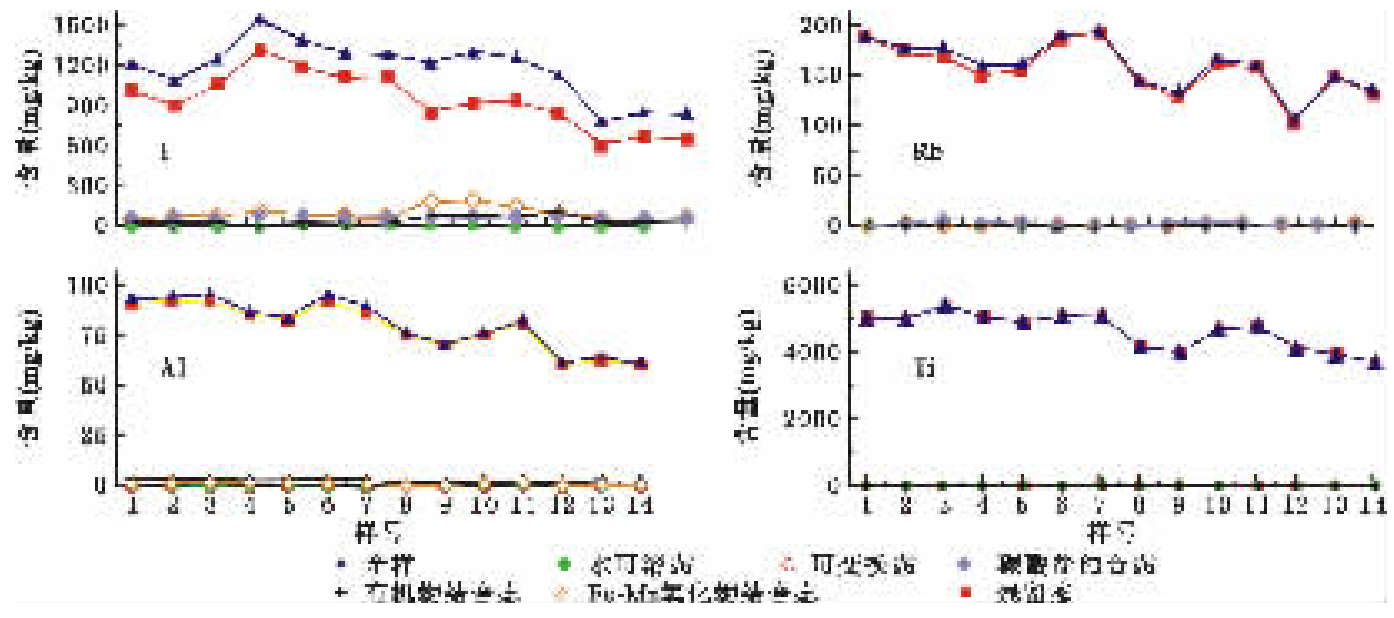

图 2 岱海沉积物中 $\mathrm{P} 、 \mathrm{Rb} 、 \mathrm{Al}$ 和 $\mathrm{Ti}$ 在各赋存状态中的含量变化

Fig. 2 The variations of $\mathrm{P}, \mathrm{Rb}, \mathrm{Al}$ and $\mathrm{Ti}$ concentrations in various fractions in the Daihal lake sediments

内蒙古岱海地处我国的干旱-半干旱过渡地带, 属中温带季风气候, 海拔 1230-1350 m, 多风, 冬季漫长 而寒冷, 夏季短而温暖, 年平均气温 $3.5^{\circ} \mathrm{C}$, 日照率高, 无霜期短, 年温差、日温差均较大, 降水量少 (多年平 均降水量 $424.9 \mathrm{~mm} / \mathrm{a}$ ), 年蒸发量大 $(1938 \mathrm{~mm} / \mathrm{a})$. 在这种半干旱气候条件下, 岱海流域基岩往往只经历较 弱的化学风化作用, 加之流域陡峻的山坡地形, 风化产物将快速地被带人湖盆, 出溶的元素或溶解于湖水 中, 或以各种形式赋存于沉积物中. 岱海沉积物主要由石英、长石类、云母类、伊利石、绿泥石、碳酸盐和少量 重矿物组成, 碎屑斜长石含量一般高于 $50 \%$, 表明岱海流域仍处于化学风化初期阶段 ${ }^{[11,12]}$. 连续提取结果 表明,大多数较为惰性的元素包括 $\mathrm{P} 、 \mathrm{Al} 、 \mathrm{Ti} 、 \mathrm{Rb} 、 \mathrm{~K} 、 \mathrm{Cr} 、 \mathrm{Co} 、 \mathrm{Cu}$ 等均主要赋存于沉积物的残留态内 (图 2), 进 一步反映了岱海流域发生的弱化学风化过程. 然而, 在弱风化过程中,仍有一些活动性强的元素如 $\mathrm{Ca} 、 \mathrm{Sr}$ 被 溶解进人溶液, 人湖后溶解于湖水或赋存在其他相态中, 还有一些元素如 $\mathrm{V} 、 \mathrm{~K} 、 \mathrm{Zn} 、 \mathrm{Mn} 、 \mathrm{Be} 、 \mathrm{~Pb} 、 \mathrm{Fe} 、 \mathrm{Cu} 、 \mathrm{Co}$ 等 发生不同程度的风化迁移. 在此,我们根据风化作用对岱海流域元素溶解迁移的程度将所测元素进一步划 分为受风化程度制约的、部分受风化程度制约的、基本不受风化程度制约的和完全不受风化程度制约的四 
种类型 (表 2).

出露于岱海流域内的第三纪玄武岩和太古代变质侵 人岩中的 $\mathrm{Sr}$ 主要以 $\mathrm{Ca}$ 的类质同像形式赋存于斜长石 内, 而斜长石是容易被风化的含岩主矿物, 因此在化学风 化过程中将造成大量 $\mathrm{Ca}^{2+} 、 \mathrm{Sr}^{2+}$ 的淋溶进人溶液被带人 湖盆内. 人湖后, $\mathrm{Sr}$ 和 $\mathrm{Ca}$ 主要赋存于碳酸盐结合态和有 机物结合态中 (图 4), 两者在水可溶态和有机物结合态 中具有很好的相关性 (图 5a,b), 这是由它们相似的地球 化学性状决定的. 由此表明, 岱海流域岩石风化溶解出的 $\mathrm{Sr}$ 和 $\mathrm{Ca}$ 是等当量的, 出溶的 $\mathrm{Sr}$ 和 $\mathrm{Ca}$ 在沉积物中主要赋 存于以自生方解石、白云石为主的自生碳酸盐矿物中或 黏附于有机物表面. 并且, 碳酸盐结合态和有机物结合态 中 $\mathrm{Sr}$ 含量的高低与化学风化强度密切相关. 我们的研究 已表明 ${ }^{[11]}$, 湖泊沉积物的高 $\mathrm{Rb} / \mathrm{Sr}$ 比值可以很好地反映 流域所经历的化学风化过程: 湖泊沉积物的 $\mathrm{Rb} / \mathrm{Sr}$ 比越 小, 流域经历的化学风化程度越强; 反之则弱. 各结合态 中 $\mathrm{Rb}$ 含量的变化表明, 在化学风化过程中 $\mathrm{Rb}$ 表现为惰

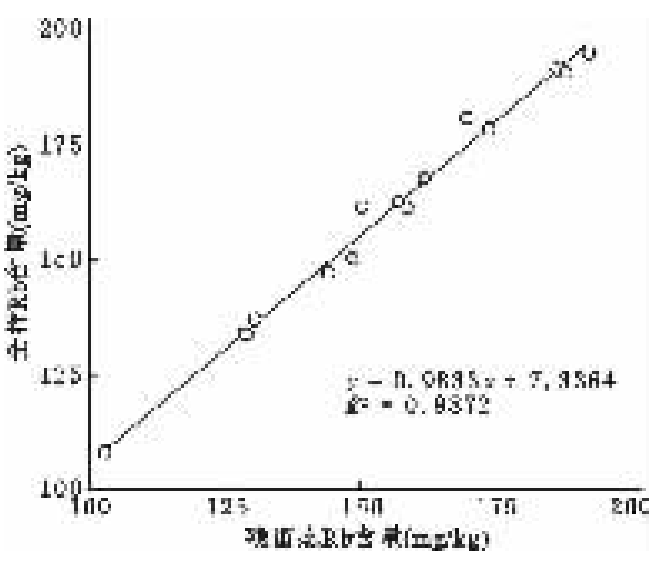

图 3 岱海沉积物中残留态中 $\mathrm{Rb}$ 含量 和全样 $\mathrm{Rb}$ 含量的正相关性

Fig. 3 A positive correlation of $\mathrm{Rb}$ concentrations in bulk and in residual fractions in the Daihai lake sediments 性, 主要赋存于残留态中 (图 2), 沉积物全样的 $\mathrm{Rb}$ 含量与残留态的 $\mathrm{Rb}$ 含量具有很好的线性相关 (图 3 ), 因 此本连续提取实验从本质上证实了岱海湖泊沉积物的 $\mathrm{Rb} / \mathrm{Sr}$ 比值变化主要受 $\mathrm{Sr}$ 含量变化制约的. 从图 4 可以看出, 沉积物、碳酸盐结合态及有机物结合态中 $\mathrm{Sr}$ 的高含量是与沉积物的低 $\mathrm{Rb} / \mathrm{Sr}$ 比值相对应的, 反 映碳酸盐结合态和有机物结合态中 $\mathrm{Sr}$ 的含量是受化学风化强度控制的; 反之碳酸盐和有机物两种结合态 中 $\mathrm{Sr}$ 含量高低可以间接地反映化学风化程度. 我们进一步的研究表明 ${ }^{[12]}$, 湖水与表层沉积物中碳酸盐具 有相同的 ${ }^{87} \mathrm{Sr} /{ }^{86} \mathrm{Sr}$ 比值, 因此自生碳酸盐元素及其同位素组成可以反映了碳酸盐形成时湖水的化学及湖区 气候环境状况.

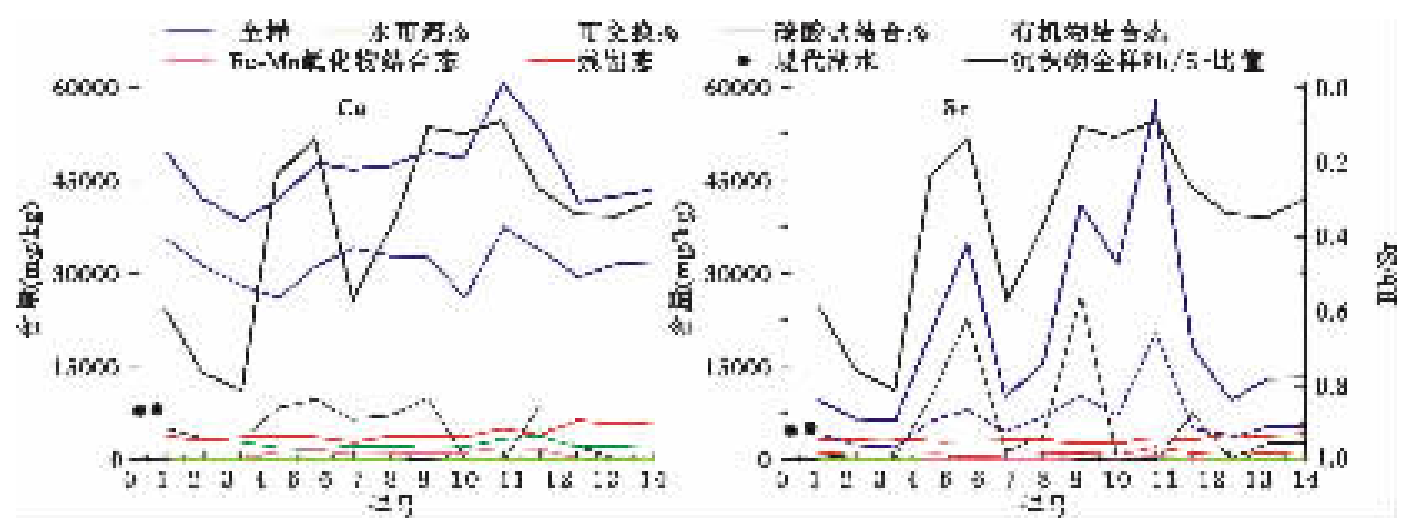

图 4 岱海沉积物 $\mathrm{Rb} / \mathrm{Sr}$ 比值、 $\mathrm{Sr}$ 和 $\mathrm{Ca}$ 在各赋存状态中的含量变化及现代湖水中 $\mathrm{Sr}$ 和 $\mathrm{Ca}$ 的含量

Fig. 4 The variations of $\mathrm{Sr}$ and Ca concentrations both in today Daihai lake water and in various fractions, and of the $\mathrm{Rb} / \mathrm{Sr}$ ratios in the Daihai lake sediments

虽然沉积物中 $\mathrm{Ca}$ 的含量与碳酸盐结合态中的 $\mathrm{Ca}$ 含量变化基本一致, 然而 $\mathrm{Ca}$ 含量的变化与反映化学 风化的 $\mathrm{Rb} / \mathrm{Sr}$ 比值并没有显著的相关性 (图 4), 表明岱海沉积物 $\mathrm{Ca}$ 含量变化并不完全受化学风化强度的 制约, 而可能与湖水的物理化学状态有关, 这与 $\mathrm{Sr}$ 的地球化学性状不同. 因此湖泊沉积物 $\mathrm{CaCO}_{3}$ 含量的变 化主要反映水文的变化状态. 高 $\mathrm{CaCO}_{3}$ 含量常常被认为是暖干的气候环境 ${ }^{[13]}$, 然而这似乎与低 $\mathrm{Rb} / \mathrm{Sr}$ 沉积 
物 (图 4) 所反映的有利于强化学风化的气候条件相矛盾. 因此, 简单地根据高 $\mathrm{CaCO}_{3}$ 含量就判断当时所处 为暖干的气候环境可能不合适, 如因化学风化增强导致湖水 $\mathrm{Ca}^{2+}$ 增高、pH 升高而形成的弱碱性的湖水环 境将有助于 $\mathrm{CaCO}_{3}$ 沉淀,但流域可能处于有利于化学风化的湿润气候条件.
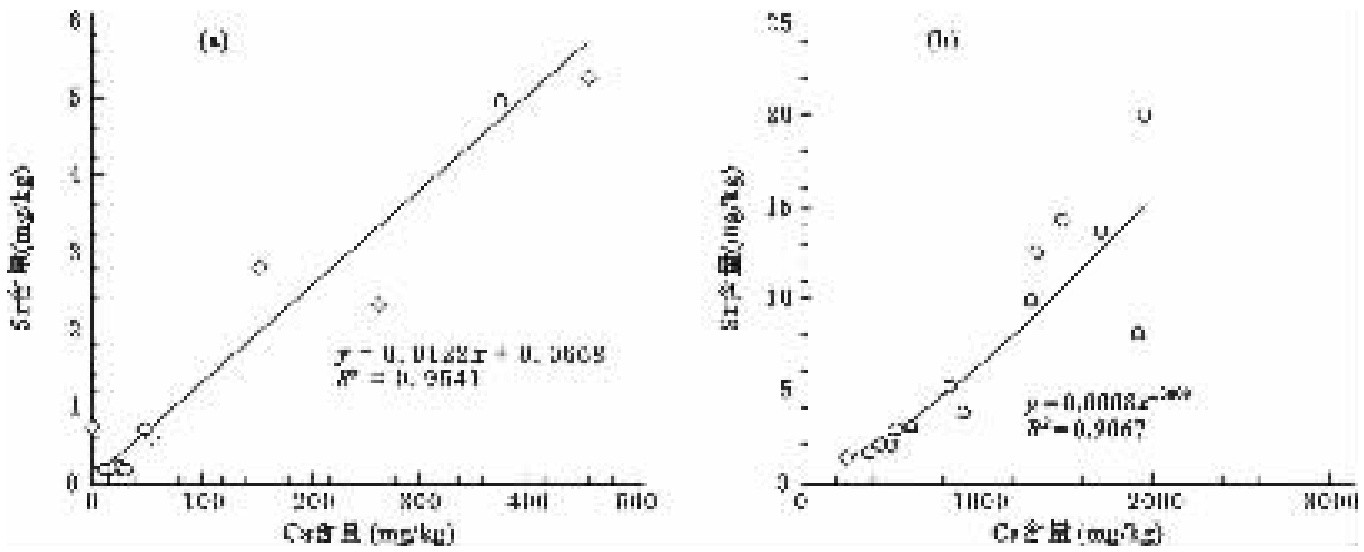

图 5 岱海沉积物中 $\mathrm{Sr}$ 和 $\mathrm{Ca}$ 在水可溶态 (a) 和有机物结合态 $(\mathrm{b})$ 中的含量的相关性

Fig. 5 Relationship between Ca and Sr concentrations in fractions of soluble (a) and organic (b) in Daihai lake sediments
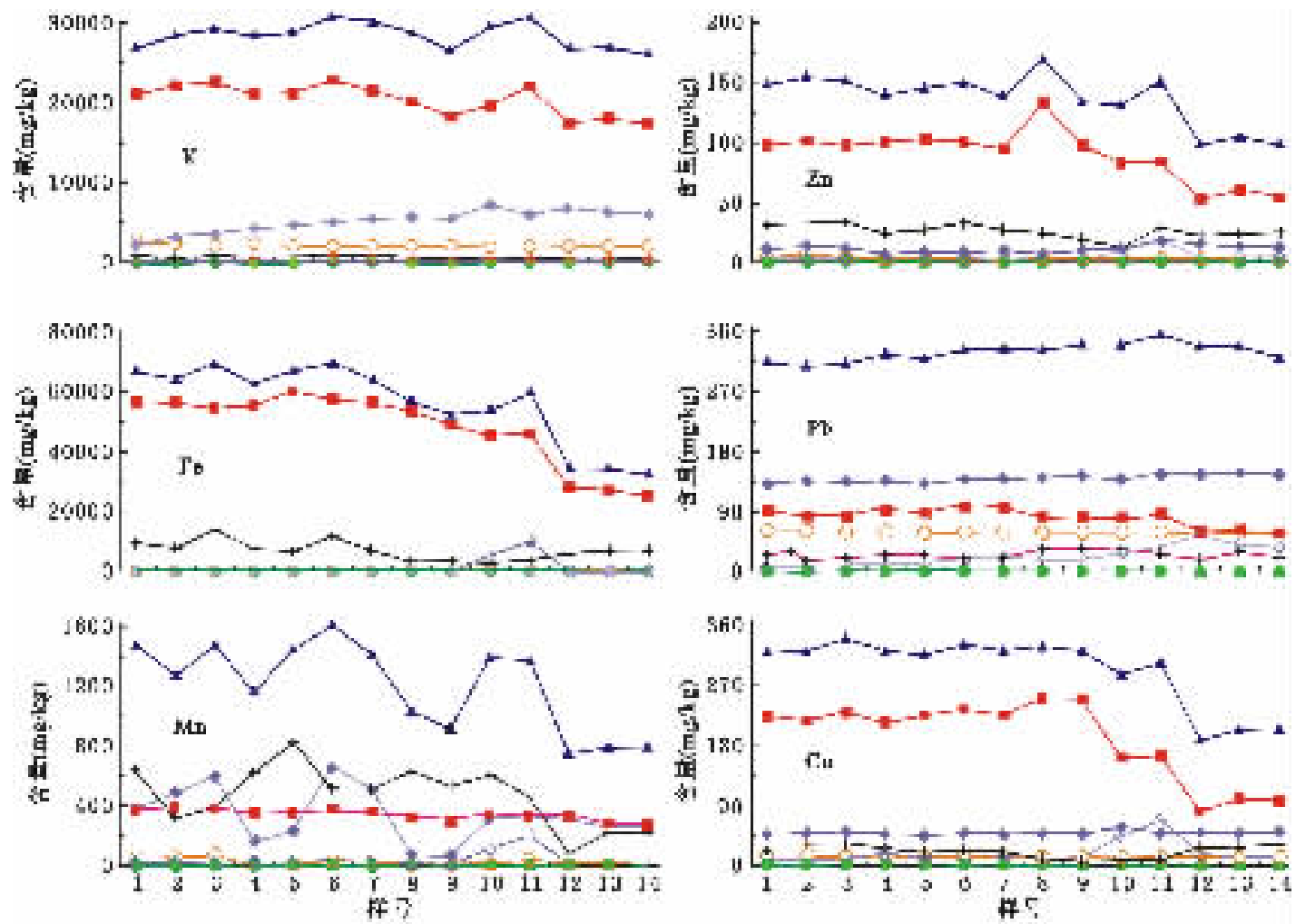

a址接

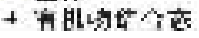

$x=\frac{x}{2}$

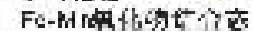

5 走走占

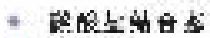

-

图 6 岱海沉积物中 $\mathrm{K} 、 \mathrm{Fe} 、 \mathrm{Mn} 、 \mathrm{Cu} 、 \mathrm{~Pb}$ 和 $\mathrm{Zn}$ 在各赋存状态中的含量变化 (图例同图 2)

Fig. 6 The variations of $\mathrm{K}, \mathrm{Fe}, \mathrm{Mn}, \mathrm{Cu}, \mathrm{Pb}$ and $\mathrm{Zn}$ concentrations in various fractions in Daihai lake sediments 


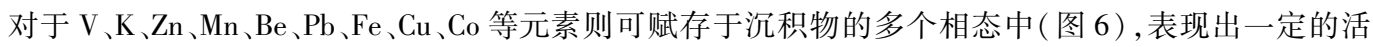
动性, 但残留态仍是主要的赋存形态. 其中, 直接从湖水结晶沉淀的碳酸盐对 $\mathrm{Pb} 、 \mathrm{Be} 、 \mathrm{Co} 、 \mathrm{Cu} 、 \mathrm{~K} 、 \mathrm{Mn}$ 等元素 赋存相态有一定的制约作用,如碳酸盐结合态是 $\mathrm{Pb} 、 \mathrm{Cu} 、 \mathrm{~K}$ 等元素非残渣相的主要赋存相态. 而 $\mathrm{Fe} 、 \mathrm{Mn} 、 \mathrm{Zn}$ 等元素则与有机质有一定的亲合性, 可形成相对稳定的且不易为生物所吸收的有机络合物或螯合物 ${ }^{[14]}$, $\mathrm{Mn}$ 在其中的含量可高达 $40 \%-50 \%$.

\section{3 结语}

流域岩石在一定气候条件下经风化剥蚀作用使元素以各种形式带人湖盆, 随后或溶解于湖水中, 或以 一定的形式赋存于沉积物的不同相态中,因此一定时段沉积物中不同赋存相态内元素含量的变化可以反映 流域经历化学风化的强度及湖水的化学组成. 元素在沉积物中的赋存相态与表生过程中元素的活动性密切 相关, 如惰性的元素 (如 $\mathrm{Ti} 、 \mathrm{Al} 、 \mathrm{Rb}$ 等)往往赋存于残留态中, 而活泼的元素则在出溶后赋存于与特定元素亲 合性强的其他相态中, 它是受特定湖盆湖水物理、化学、生物等状态制约的,如 $\mathrm{Fe} 、 \mathrm{Mn} 、 \mathrm{Zn}$ 等元素主要赋存 于氧化物结合态和有机质结合态中, 而 $\mathrm{Pb} 、 \mathrm{Cu} 、 \mathrm{~K}$ 等元素非残渣相的主要赋存相态是自生碳酸盐. 在此, 本 连续提取实验结果为评价单一流域表生过程及湖泊沉积物中元素赋存相态、化学变化提供了有力的地球化 学依据. 同时,在运用沉积物中元素含量或比值的变化来解释气候环境变化状态时,需要综合考虑流域和水 体两个体系对元素地球化学行为完全不同的制约行为.

致谢: $\mathrm{Rb}$ 的含量在南京大学现代分析中心测定; 其它元素含量测定在南京大学成矿作用国家重点实验室完 成. 同济大学刘连文博士在实验方面给予协助, 并与作者进行了有益的探讨; 南京大学装丽雯老师和本所的 隋桂荣老师在实验室工作和分析方面给予了大力的协作,在此作者一并表示感谢.

\section{4 参考文献}

[1] Li Y H. Distribution patterns of elements in the ocean: A synthesis. Geochim Cosmochim Acta, 1991,55 : 3223 - 3240.

[2] Stoll H M, Schrag D P. Sr/Ca variations in Cretaceous carbonates: relation to productivity and sea level changes. Palaeogeogr Palaeoclim Palaeoeco,2001, 168: 311 - 336.

[3] 曹建廷,沈吉,金章东. 内蒙古岱海地区小冰期气候特征的地球化学记录. 南京师范大学学报, 2000,23 : 247 -251 .

[4] Rognerud S, Fjeld E. Trace element contamination of Norwegian lake sediments. Ambio, 2001, 30 : $11-19$.

[5] Lithner G, Borg H, Elk J, et al. The turnover of metals in a eutrophic and an oligotrophic lake in Sweden. Ambio, 2000, 29: $216-228$.

[6] Tessier A, Campbell P G C, Bisson M. Sequential extraction procedure for the speciation of particulate trace metals. Analytical Chemistry, 1979, 51: $844-851$.

[7] Li X, Coles B J, Ramsey M H, et al. Sequential extraction of soils for multi-element analysis by ICP-AES. Chem Geol, $1995,124: 109-123$.

[8] Khebonian C, Bauer C. Accuracy of selective extraction procedures for metal speciation in model aquatic sediments. Analytical Chemistry, 1987, 59: 1417 - 1423.

[9] 陈 骏, 王洪涛, 鹿化显. 陕西洛川黄土沉积物中稀土元素及其它微量元素的化学淋滤研究. 地质学报, 1996, $70: 61-72$.

[10] 赵志琦, 刘丛强, 肖应凯. 黄土 - 古土壤沉积物中酸溶相硼的分离及其同位素测定. 地球化学, $2000,29: 351-357$.

[11] 金章东, 王苏民, 沈 吉等. 小冰期弱化学风化的湖泊沉积记录. 中国科学( D 辑), 2001, 31: $221-225$.

[12] 金章东, 王苏民, 沈 吉等. 湖泊沉积物 Sr 同位素记录的小冰期. 科学通报, 2002, 47: 1512-1516.

[13] Zhong W, Wang J. Preliminary study of Holocene environmental changes in Xinjiang. Chinese Geography Science, 1996, 6: $166-175$.

[14] Fernandes H M. Heavy metal distribution in sediments and ecological risk assessment: the role of diagenetic processes in reducing metal toxicity in bottom sediments. Environmental Pollution, 1997, 97: 317 - 325. 